\title{
Photon decaying in de Sitter universe
}

\author{
Y. Ahmadi ${ }^{1}$ M. V. Takook ${ }^{2}$
}

Received: 20 August 2019 / Accepted: 25 September 2019 / Published online: 8 October 2019

(c) The Author(s) 2019

\begin{abstract}
The interaction between three photons is studied in de Sitter ambient space formalism. As a special case, the half harmonic generator is considered, i.e., one photon decays to two same-energy photons. The scattering matrix elements are presented which define the indirect gravitational effect on quantum field theory. The null curvature limit of scattering matrix is obtained for comparing it with its Minkowskian counterpart. The Hamiltonian of this interaction, in Minkowski space-time, was presented by using the quantum vacuum fluctuation in the one-loop approximation.
\end{abstract}

Keywords de Sitter space-time $\cdot$ Interaction $\cdot$ Quantum field theory $\cdot$ Scattering matrix

\section{Introduction}

Historically, the interaction of light with matter is one of the most important interactions in physics. This interaction plays an important role in technology, which can be explained completely in quantum field theory (QFT) model. In quantum level, the interaction between photons, i.e., wave mixing, has a special role in quantum optics such as fiber laser and squeezed light. The process of decaying one photon to two photons is named as second harmonic generator (SHG). The reverse of this process, i.e., merging two photons and exiting one photon, is also possible. These interactions are performed by using some crystals. In flat space, SHG is used for generating the coherent state [1-4]. In quantum optics, the Hamiltonian of SHG process can be written as $\mathcal{H}=\hbar \chi\left(d^{\dagger} d^{\prime} d^{\prime \prime}+d d^{\prime \dagger} d^{\prime \prime \dagger}\right)[3,5]$. The $d$ and $d^{\dagger}$ are annihilation and creation operators, respectively, and $\chi$ is interaction constant which is fixed phenomenologically $[1-3,6$, 7]. The $\chi$ constant can be obtained from QFT point of view which is studied in [43] and recall in section "Scattering matrix in null curvature limit".

In curved space-time, the interaction of fields is affected by background gravitational field. By computing the

\footnotetext{
M. V. Takook

takook@razi.ac.ir

Y. Ahmadi

ahmadi.pave@gmail.com

1 Department of Physics, Razi University, Kermanshah, Iran

2 Department of Physics, Kharazmi University, Tehran, Iran
}

scattering matrix, this indirect gravitational effects can be seen. In previous papers, the interaction between vector and spinor fields and also the interaction between scalar and spinor fields are considered and the scattering matrix is calculated $[8,9]$. By attention to that the experimental data it is clear that the our universe and early universe can be described by de Sitter (dS) space-time [10-15] so the construction of quantum field theory (QFT) in dS space-time is important for quantum gravity and unified theory. Some efforts have been made for constructing QFT in $\mathrm{dS}$ ambient space formalism in the last few years [16-26]. One of the advantages of ambient space formalism is the simplicity in calculations of probability amplitude because the action of $\mathrm{dS}$ group on ambient space coordinate is linear [22]. In this paper, the decaying of one photon to two same-energy photons in $\mathrm{dS}$ ambient space formalism is investigated and the scattering matrix of this interaction is obtained in the first approximation. Finally, its null curvature limit is discussed.

The organization of this article is as follows. The notation and terminology that have been used in this article are presented in "Notation" section. The $\mathcal{S}$-matrix elements in the small-curvature approximation are investigated in $\mathrm{dS}$ ambient space formalism in "Scattering matrix in dS space-time" section. In "Scattering matrix in null curvature limit" section, the decaying of one photon to two same-energy photons has been recalled in Minkowski space-time. In Section V, we calculate the null curvature limit of the $\mathcal{S}$-matrix elements and compare it with Minkowskian counterpart. Finally, the conclusion has been presented in Section VI. 


\section{Notation}

The dS space-time can be considered as a four-dimensional hyperboloid embedded in five-dimensional Minkowski space-time:

$$
\begin{gathered}
M_{H}=\left\{x \in \mathbb{R}^{5} \mid x \cdot x=\eta_{\alpha \beta} x^{\alpha} x^{\beta}=-H^{-2}\right\}, \\
\alpha, \beta=0,1,2,3,4 .
\end{gathered}
$$

The metric is:

$$
\begin{gathered}
\mathrm{d} s^{2}=\left.\eta_{\alpha \beta} \mathrm{d} x^{\alpha} \mathrm{d} x^{\beta}\right|_{x^{2}=-H^{-2}}=g_{\mu \nu}^{\mathrm{d} S} \mathrm{~d} X^{\mu} \mathrm{d} X^{\nu}, \\
\mu, \nu=0,1,2,3,
\end{gathered}
$$

where $\eta_{\alpha \beta}=\operatorname{diag}(1,-1,-1,-1,-1), H$ is Hubble constant parameter, $X^{\mu}$ is dS intrinsic coordinate and $x^{\alpha}$ is the fivedimensional dS ambient space formalism.

The action of dS group, $S O(1,4)$, on the intrinsic coordinate $X^{\mu}$ is complicated but the action of this group on the ambient space coordinate $x^{\alpha}$ is linear. So the calculation in this formalism is simple and similar to Minkowski space-time [22]. In this formalism, the five matrices $\gamma^{\alpha}$ are needed which satisfy the following relations [16, 20, 22, 27]:

$\gamma^{\alpha} \gamma^{\beta}+\gamma^{\beta} \gamma^{\alpha}=2 \eta^{\alpha \beta}, \gamma^{\alpha \dagger}=\gamma^{0} \gamma^{\alpha} \gamma^{0}$,

and they can be chosen as:

$$
\begin{aligned}
& \gamma^{0}=\left(\begin{array}{cc}
I & 0 \\
0 & -I
\end{array}\right), \quad \gamma^{4}=\left(\begin{array}{cc}
0 & I \\
-I & 0
\end{array}\right), \gamma^{1}=\left(\begin{array}{cc}
0 & i \sigma^{1} \\
i \sigma^{1} & 0
\end{array}\right), \\
& \gamma^{2}=\left(\begin{array}{cc}
0 & -i \sigma^{2} \\
-i \sigma^{2} & 0
\end{array}\right), \quad \gamma^{3}=\left(\begin{array}{cc}
0 & i \sigma^{3} \\
i \sigma^{3} & 0
\end{array}\right),
\end{aligned}
$$

where $\sigma^{i}(i=1,2,3)$ are the Pauli matrices. The $\gamma^{\alpha}$ matrices in $\mathrm{dS}$ ambient space formalism are different from Minkowski $\gamma^{\prime \mu}$ matrices. The relation between them is [27]:

$\gamma^{\prime \mu}=\gamma^{\mu} \gamma^{4}$.

The dS-Dirac first-order field equation is [16, 22]:

$\left(\not x \not \partial^{\top}-2 \pm i v\right) \Psi(x)=0, \quad \not x=\gamma_{\alpha} x^{\alpha}=\gamma \cdot x$,

where $v>0$ is related to $\mathrm{dS}$ mass parameter as $m_{f, v}^{2}=H^{2}\left(v^{2}+2 \pm i v\right)$ and $\partial_{\alpha}^{\top}=\partial_{\alpha}+H x_{\alpha} x . \partial$ is the transverse derivative. The charged spinor field operator, which satisfies the field equation (2.5), is [22, 27]:

$$
\begin{aligned}
\Psi(x) & =\mathcal{N}_{p} \int \mathrm{d} \mu(\xi) \sum_{\sigma= \pm \frac{1}{2}}\left[a(\tilde{\xi}, \sigma)(H x . \xi)^{-2-i v} \mathcal{U}(x, \xi, \sigma)\right. \\
& \left.+b^{\dagger}(\xi, \sigma)(H x . \xi)^{-1+i v} \mathcal{V}(x, \xi, \sigma)\right],
\end{aligned}
$$

where $\mathcal{N}_{p}$ is normalization constant and $\xi^{\alpha}=\left(\xi^{0}, \vec{\xi}, \xi^{4}\right) \in C^{+}=\left\{\xi \in \mathbb{R}^{5} \mid \xi \cdot \xi=0, \quad \xi^{0}>0\right\}$ is the transformed variable of $x^{\alpha}$ in positive cone. The $\xi^{\alpha}$ becomes the energy-momentum four-vector in null curvature limit. Also the $\tilde{\xi}$ is $\tilde{\xi}^{\alpha}=\left(\xi^{0},-\vec{\xi}, \xi^{4}\right)$ and the $\mathrm{d} \mu(\xi)$ is the $S O(4)-$ invariant normalized volume. The explicit form of $\mathcal{U}$ and $\mathcal{V}$ is presented in [27]. $a^{\dagger}(\xi, \sigma)$ and $b^{\dagger}(\xi, \sigma)$ are creation operators which act on $\mathrm{dS}$-invariant vacuum sate $|\Omega\rangle$ as: [22]:

$a^{\dagger}(\xi, \sigma)|\Omega\rangle \equiv\left|1_{\xi, \sigma}^{a}\right\rangle, \quad b^{\dagger}(\xi, \sigma)|\Omega\rangle \equiv\left|1_{\xi, \sigma}^{b}\right\rangle$.

The anti-commutation relations for creation and annihilation operators are:

$\left\{a\left(\tilde{\xi}^{\prime}, \sigma^{\prime}\right), a^{\dagger}(\xi, \sigma)\right\}=\delta^{3}\left(\xi-\xi^{\prime}\right) \delta_{\sigma \sigma^{\prime}}$,

$\left\{b\left(\tilde{\xi}^{\prime}, \sigma^{\prime}\right), b^{\dagger}(\xi, \sigma)\right\}=\delta^{3}\left(\xi-\xi^{\prime}\right) \delta_{\sigma \sigma^{\prime}}$.

Analytic field operator is defined in complex dS space-time as [22, 29]:

$\Psi(x)=\lim _{y \rightarrow 0} \Psi(z)=\lim _{y \rightarrow 0} \Psi(x+i y)$.

The adjoint spinor $\bar{\Psi}(x)$ in ambient space formalism which is defined as $\bar{\Psi}(x)=\Psi^{\dagger}(x) \gamma^{0} \gamma^{4}$ satisfies this field equation [16, 20, 22, 27, 28]:

$\bar{\Psi}(x) \gamma^{4}\left(\overleftarrow{\partial}^{\top} \not x-2 \mp i v\right)=0$

The analytic two-point function of spinor field is [22, 27]:

$$
\begin{aligned}
& \left\langle\Omega\left|\Psi_{i}\left(z_{1}\right) \bar{\Psi}_{j}\left(z_{2}\right)\right| \Omega\right\rangle=i \mathbf{S}_{i j}\left(z_{1}, z_{2}\right) \\
& =\frac{c_{\frac{1}{2}, v}}{2} \int_{S^{3}} \mathrm{~d} \mu(\xi)\left(z_{1} \cdot \xi\right)^{-2-i v}\left(z_{2} \cdot \xi\right)^{-2+i v}\left(\xi \gamma^{4}\right)_{i j},
\end{aligned}
$$

where $c_{\frac{1}{2}, v}$ is the normalization constant. This analytic twopoint function can be calculated in terms of generalized Legendre function of first kind [16, 20, 27].

As it is discussed in $\mathrm{V}$, the null curvature limit of the quantum field operator of spinor field $\Psi(x)$ reduces to its Minkowski counterpart $\psi(X)$. In Minkowski space-time, the Dirac equation is $(i \not \partial-m) \psi=0$. In this equation, $\not \partial=\gamma^{\prime} \mu \partial_{\mu}$ and $\gamma^{\prime \mu}$ matrices are $4 \times 4$ matrices which satisfy the following conditions [30-33]:

$\gamma^{\prime \mu} \gamma^{\prime \nu}+\gamma^{\prime \nu} \gamma^{\prime \mu}=2 \eta^{\mu \nu}, \quad \gamma^{\prime \mu \dagger}=\gamma^{\prime 0} \gamma^{\prime \mu} \gamma^{\prime 0}$,

where the $\eta_{\mu \nu}$ is Minkowski metric.

In the ambient space formalism, one can write the massless vector field, similar to other massless quantum field, in terms of the massless conformally coupled scalar field. The field operator is obtained in terms of annihilation $d$ and creation $d^{\dagger}$ operators as [16, 34]:

$$
\begin{aligned}
\mathcal{A}_{\alpha}(x) & =\mathcal{N}_{k} \int \mathrm{d} \mu(\xi)_{B} \sum_{n}\left[d(\tilde{\xi}, n)(H x . \xi)^{-2} \mathcal{E}_{2 \alpha}(x, \xi, n)\right. \\
& \left.+d^{\dagger}(\xi, n)(H x . \xi)^{-1} \mathcal{E}_{1 \alpha}(x, \xi, n)\right],
\end{aligned}
$$


where the $\mathcal{N}_{k}$ is the normalization constant, $n=0,1,2,3$ are the polarization states and the $\mathrm{d} \mu_{B}(\xi)$ is $\mathrm{d} \mu(\xi)_{B}=2 \pi^{2} r^{3} \mathrm{~d} r \mathrm{~d} \mu(\xi)[16,35]$. Also $\mathcal{E}_{2 \alpha}$ and $\mathcal{E}_{1 \alpha}$ are polarization vectors which are studied in [16, 34]. The commutation relation between creation and annihilation operators and their action on vacuum state $|\Omega\rangle$ are $[34,36,37]$ :

$$
\begin{aligned}
& {\left[d\left(\tilde{\xi}^{\prime}, n^{\prime}\right), d^{\dagger}(\xi, n)\right]=\delta_{s^{3}}\left(\xi-\xi^{\prime}\right) \delta_{n n^{\prime}},} \\
& d(\tilde{\xi}, n)|\Omega\rangle=0, \quad d^{\dagger}(\xi, n)|\Omega\rangle=\left|1_{\xi, n}^{d}\right\rangle .
\end{aligned}
$$

The vector field operator can be written as two "positive" and "negative" parts as:

$$
\begin{aligned}
& x^{\alpha} \equiv\left(\sqrt{H^{-2}-r^{2}} \sinh H t_{s}, \sqrt{H^{-2}-r^{2}} \cosh H t_{s},\right. \\
&r \cos \theta, r \sin \theta \cos \phi, r \sin \theta \sin \phi),
\end{aligned}
$$

w h e r e $\quad-\infty<t_{s}<\infty, 0 \leq r<H^{-1}, 0 \leq$ $\theta \leq \pi, 0 \leq \phi<2 \pi$. This coordinate system does not cover all dS hyperboloids. The time evolution operator for a quantum dS black hole was considered in this coordinate system [39]. But generally, in curved space-time, it is very complicated or impossible to calculate the $\mathcal{S}$-matrix elements in $\mathrm{dS}$ space due to the event horizon [39-41]. In this work, because of the occurrence of interaction in atomic dimen-

$\mathcal{A}_{\alpha}=\mathcal{A}_{\alpha}^{(+)}+\mathcal{A}_{\alpha}^{(-)} ;\left\{\begin{array}{l}\mathcal{A}_{\alpha}^{(+)}(x)=\mathcal{N}_{k} \int \mathrm{d} \mu(\xi)_{B} \sum_{n} d(\tilde{\xi}, n)(H x . \xi)^{-2} \mathcal{E}_{2 \alpha}(x, \xi, n), \\ \mathcal{A}_{\alpha}^{(-)}(x)=\mathcal{N}_{k} \int \mathrm{d} \mu(\xi)_{B} \sum_{n} d^{\dagger}(\xi, n)(H x . \xi)^{-1} \mathcal{E}_{1 \alpha}(x, \xi, n) .\end{array}\right.$

The null curvature limit of dS quantum operator of vector field in ambient space formalism is matched on Minkowski quantum vector field operator $A_{\mu}(X)$.

\section{Scattering matrix in dS space-time}

In ambient space formalism of dS space-time, the dS-Dirac field equation is invariant under $U(1)$ global symmetry, but it is not invariant under the local $U(1)$ symmetry. By changing the gauge covariant derivative $D_{\alpha}=\partial_{\alpha}^{\top}+i q \mathcal{A}_{\alpha}$, with derivative $\partial_{\alpha}^{\top}$, one can obtain dS-Dirac local gauge invariant equation [38]. By applying this change in the free-field electromagnetic Lagrangian, the interaction Lagrangian can be obtained as [38]:

$$
\begin{aligned}
\mathcal{L}_{0} & =H \bar{\Psi}(x) \gamma^{4}\left(-i \not x \partial^{\top}+2 i+v\right) \Psi(x), \\
\mathcal{L}_{\text {int }} & =q H \bar{\Psi}(x) \gamma^{4} \not \supset \mathcal{A}(x) \Psi(x) .
\end{aligned}
$$

The $q$ in the null curvature limit can be considered as the electric charge $e$. As it is seen, one can write:

$\mathcal{H}_{\text {int }}=-\mathcal{L}_{\text {int }}=-q H \bar{\Psi}(x) \gamma^{4} \not y \mathcal{A}(x) \Psi(x)$.

In $\mathrm{d} S$ space-time, the time evolution operator, $|\alpha, t\rangle=U\left(t, t_{0}\right)\left|\alpha, t_{0}\right\rangle$, is only defined in the static coordinate system:

$$
\begin{aligned}
& \overline{\mathcal{S}^{(3)}=\frac{i}{6 \hbar^{3}} \iiint \mathrm{d} \mu\left(x_{1}\right) \mathrm{d} \mu\left(x_{2}\right) \mathrm{d} \mu\left(x_{3}\right) T\left[\mathcal{H}\left(x_{1}\right) \mathcal{H}\left(x_{2}\right) \mathcal{H}\left(x_{3}\right)\right]} \\
& =-\frac{i q^{3}}{6 \hbar^{3}} \iiint \mathrm{d} \mu\left(x_{1}\right) \mathrm{d} \mu\left(x_{2}\right) \mathrm{d} \mu\left(x_{3}\right) T\left[\bar{\Psi}_{i}\left(x_{1}\right)\left(\gamma^{4} H \not \not_{1}\right)_{i l} \mathcal{A}_{l j}\left(x_{1}\right) \Psi_{j}\left(x_{1}\right)\right. \\
& \left.\times \bar{\Psi}_{i^{\prime}}\left(x_{2}\right)\left(\gamma^{4} H \not y_{2}\right)_{i^{\prime} l^{\prime}} \mathcal{A}_{l^{\prime j^{\prime}}}\left(x_{2}\right) \Psi_{j}^{\prime}\left(x_{2}\right) \bar{\Psi}_{i^{\prime \prime}}\left(x_{3}\right)\left(\gamma^{4} H \not x_{3}\right)_{i^{\prime \prime} l^{\prime \prime}} \quad \mathcal{A}_{l^{\prime \prime} j^{\prime \prime}}\left(x_{3}\right) \Psi_{j^{\prime \prime}}\left(x_{3}\right)\right] .
\end{aligned}
$$

sion, we can ignore the direct effect of curvature in this dimension but the indirect effect of curvature exists. In this approximation, the time evolution operator can be expanded in terms of Minkowskian counterpart:

$U\left(t, t_{0}\right)=U_{M}\left(t, t_{0}\right)+H f\left(t, t_{0}\right)+\ldots$.

The $U_{M}$, in the null curvature limit, is exactly the Minkowski time evolution operator but the term $f\left(t, t_{0}\right)$ is due to the direct effect of curvature. Given that the interaction is in atomic level, one can ignore the $f\left(t, t_{0}\right)$. Although it is very difficult to calculate the scattering matrix in curved space, in atomic interaction approximation, the $\mathcal{S}$ matrix can be presented in the following approximate equation to calculate the indirect effect of curvature corrections from the Minkowski counterpart $[8,9]$ :

$$
\begin{aligned}
& \mathcal{S} \simeq \sum_{\lambda=0}^{\infty} \mathcal{S}^{(\lambda)} \\
& \mathcal{S}^{(\lambda)}=\frac{(-i)^{\lambda}}{\lambda !} \int \mathrm{d}^{4} x_{1} \cdots \int \mathrm{d}^{4} x_{\lambda} T\left[\mathcal{H}_{\text {int }}\left(x_{1}\right) \cdots \mathcal{H}_{\text {int }}\left(x_{\lambda}\right)\right] .
\end{aligned}
$$

Therefore, by inserting (3.1) in (3.2) for $\lambda=3$ and by writing the matrices in terms of their components, the $\mathcal{S}^{(3)}$ is: 
By using Wick theorem, one can write the time-order product in terms of normal-order product. The time-order product becomes to 76 normal-order products, but only two terms describe interactions $\gamma \rightarrow \gamma^{\prime}+\gamma^{\prime \prime}$ and $\gamma^{\prime}+\gamma^{\prime \prime} \rightarrow \gamma$. Therefore, by noticing (2.9), the $\mathcal{S}_{\left(\gamma \rightarrow \gamma^{\prime}+\gamma^{\prime \prime}, \gamma^{\prime}+\gamma^{\prime \prime} \rightarrow \gamma\right)}^{(3)}$ is:

$$
\begin{aligned}
& \mathcal{S}_{\left(\gamma \rightarrow \gamma^{\prime}+\gamma^{\prime \prime}, \gamma^{\prime}+\gamma^{\prime \prime} \rightarrow \gamma\right)}^{(3)} \\
& =-\frac{q^{3}}{6 \hbar^{3}} \iiint \mathrm{d} \mu\left(x_{1}\right) \mathrm{d} \mu\left(x_{2}\right) \mathrm{d} \mu\left(x_{3}\right) N\left[\mathcal{A}_{l j}\left(x_{1}\right) \mathcal{A}_{l^{\prime j^{\prime}}}\left(x_{2}\right) \mathcal{A}_{l^{\prime j^{\prime}}}\left(x_{3}\right)\right] \\
& \times\left\{\left(\gamma^{4} H x_{1}\right)_{i l} \mathbf{S}_{j j^{\prime}}\left(x_{1}, x_{2}\right)\left(\gamma^{4} H x_{2}\right)_{i^{\prime} l^{\prime}} \mathbf{S}_{j^{\prime i^{\prime \prime}}}\left(x_{2}, x_{3}\right)\left(\gamma^{4} H x_{3}\right)_{i^{\prime \prime} l^{\prime}} \mathbf{S}_{j^{\prime \prime} i}\left(x_{3}, x_{1}\right)\right. \\
& \left.+\left(\gamma^{4} H x_{1}\right)_{i l} \mathbf{S}_{j i^{\prime \prime}}\left(x_{1}, x_{3}\right)\left(\gamma^{4} H x_{3}\right)_{i^{\prime \prime} l^{\prime \prime}} \mathbf{S}_{j^{\prime \prime} i^{\prime}}\left(x_{3}, x_{2}\right)\left(\gamma^{4} H x_{2}\right)_{i^{\prime \prime} l^{\prime}} \mathbf{S}_{j^{\prime} i}\left(x_{2}, x_{1}\right)\right\} \text {, }
\end{aligned}
$$

Two terms of (3.4) describe the diagrams with counterclockwise and anticlockwise spinor loops shown in Fig. 1.

The second term in (3.4) by exchanging $x_{1}$ and $x_{2}$ becomes the first term, so one can consider one of them. Therefore,

$$
\begin{aligned}
& \mathcal{S}_{\left(\gamma \rightarrow \gamma^{\prime}+\gamma^{\prime \prime}\right)}^{(3)} \\
& =i \iiint \mathrm{d} \mu\left(\xi_{k}\right) \mathrm{d} \mu\left(\xi_{k^{\prime}}\right) \mathrm{d} \mu\left(\xi_{k^{\prime \prime}}\right) \\
& \quad \sum_{r} \sum_{r^{\prime}} \sum_{r^{\prime \prime}} \chi^{\mathrm{d} S} a^{r}(k) a^{\dagger r^{\prime}}\left(k^{\prime}\right) a^{\dagger r^{\prime \prime}}\left(k^{\prime \prime}\right),
\end{aligned}
$$

where

$$
\begin{aligned}
& \chi^{\mathrm{d} S}=\frac{i q^{3}}{\hbar^{3}} \mathcal{N}_{k} \mathcal{N}_{k^{\prime}} \mathcal{N}_{k^{\prime \prime}} \\
& \iiint \mathrm{d} \mu\left(x_{1}\right) \mathrm{d} \mu\left(x_{2}\right) \mathrm{d} \mu\left(x_{3}\right)\left(H x_{1} \cdot \xi_{k}\right)^{-2}\left(H x_{2} \cdot \xi_{k^{\prime}}\right)^{-1}\left(H x_{3} \cdot \xi_{k^{\prime \prime}}\right)^{-1} \\
& \times \operatorname{Tr}\left\{\gamma^{4} H x_{1} q_{2} \mathbf{S}\left(x_{1}, x_{2}\right) \gamma^{4} H x_{2} q_{1} \mathbf{S}\left(x_{2}, x_{3}\right) \gamma^{4} H x_{3} \mathcal{q}_{1} \mathbf{S}\left(x_{3}, x_{1}\right)\right\} .
\end{aligned}
$$

In (3.6), one can write the $\mathbf{S}\left(x, x^{\prime}\right)$ as (2.9) or in terms of generalized Legendre function [22] and then calculate the (3.6) by numerical methods. These calculations may open

$$
\begin{aligned}
\mathcal{S}_{\left(\gamma \rightarrow \gamma^{\prime}+\gamma^{\prime \prime}, \gamma^{\prime}+\gamma^{\prime \prime} \rightarrow \gamma\right)}^{(3)} & \\
= & -\frac{q^{3}}{3 \hbar^{3}} \iiint \mathrm{d} \mu\left(x_{1}\right) \mathrm{d} \mu\left(x_{2}\right) \mathrm{d} \mu\left(x_{3}\right) N\left[\mathcal{A}_{l j}\left(x_{1}\right) \mathcal{A}_{l^{\prime} j^{\prime}}\left(x_{2}\right) \mathcal{A}_{l^{\prime \prime} j^{\prime \prime}}\left(x_{3}\right)\right] \\
& \times\left\{\left(\gamma^{4} H \not y_{1}\right)_{i l^{\prime}} \mathbf{S}_{j i^{\prime}}\left(x_{1}, x_{2}\right)\left(\gamma^{4} H \not x_{2}\right)_{i^{\prime} l^{\prime} l^{\prime}} \mathbf{S}_{j^{\prime} i^{\prime \prime}}\left(x_{2}, x_{3}\right)\left(\gamma^{4} H \not x_{3}\right)_{i^{\prime \prime} l^{\prime \prime}} \mathbf{S}_{j^{\prime \prime} i}\left(x_{3}, x_{1}\right)\right\} .
\end{aligned}
$$

By separating the $\mathcal{A}$ to positive and negative parts (2.12), one can obtain the $N\left[\mathcal{A}_{l j}\left(x_{1}\right) \mathcal{A}_{l^{\prime} j^{\prime}}\left(x_{2}\right) \mathcal{A}_{l^{\prime \prime} j^{\prime \prime}}\left(x_{3}\right)\right]$ as:

$$
\begin{aligned}
N[ & \left.\mathcal{A}_{l j}\left(x_{1}\right) \mathcal{A}_{l^{\prime} j^{\prime}}\left(x_{2}\right) \mathcal{A}_{l^{\prime \prime} j^{\prime \prime}}\left(x_{3}\right)\right] \\
= & \mathcal{A}_{l j}^{(+)}\left(x_{1}\right) \mathcal{A}_{l^{\prime} j^{\prime}}^{(+)}\left(x_{2}\right) \mathcal{A}_{l^{\prime \prime} j^{\prime \prime}}^{(+)}\left(x_{3}\right)+\mathcal{A}_{l j}^{(-)}\left(x_{1}\right) \mathcal{A}_{l^{\prime} j^{\prime}}^{(-)}\left(x_{2}\right) \mathcal{A}_{l^{\prime \prime} j^{\prime \prime}}^{(-)}\left(x_{3}\right) \\
& +\mathcal{A}_{l j}^{(+)}\left(x_{1}\right) \mathcal{A}_{l^{\prime} j^{\prime}}^{(+)}\left(x_{2}\right) \mathcal{A}_{l^{\prime \prime} j^{\prime \prime}}^{(-)}\left(x_{3}\right) \\
& +\mathcal{A}_{l j}^{(+)}\left(x_{1}\right) \mathcal{A}_{l^{\prime \prime} j^{\prime \prime}}^{(+)}\left(x_{3}\right) \mathcal{A}_{l^{\prime} j^{\prime}}^{(-)}\left(x_{2}\right)+\mathcal{A}_{l^{\prime} j^{\prime}}^{(+)}\left(x_{2}\right) \mathcal{A}_{l^{\prime \prime} j^{\prime \prime}}^{(+)}\left(x_{3}\right) \mathcal{A}_{l j}^{(-)}\left(x_{1}\right) \\
& +\mathcal{A}_{l j}^{(+)}\left(x_{1}\right) \mathcal{A}_{l^{\prime} j^{\prime}}^{(-)}\left(x_{2}\right) \mathcal{A}_{l^{\prime \prime} j^{\prime \prime}}^{(-)}\left(x_{3}\right)+\mathcal{A}_{l^{\prime} j^{\prime}}^{(+)}\left(x_{2}\right) \mathcal{A}_{l j}^{(-)}\left(x_{1}\right) \mathcal{A}_{l^{\prime \prime} j^{\prime \prime}}^{(-)}\left(x_{3}\right) \\
& +\mathcal{A}_{l^{\prime \prime} j^{\prime \prime}}^{(+)}\left(x_{3}\right) \mathcal{A}_{l j}^{(-)}\left(x_{1}\right) \mathcal{A}_{l^{\prime} j^{\prime}}^{(-)}\left(x_{2}\right) .
\end{aligned}
$$

Here, two first terms are unphysical and three terms in the second line represent the interaction $\gamma^{\prime}+\gamma^{\prime \prime} \longrightarrow \gamma$ and also three terms in the third line represent the interaction $\gamma \longrightarrow \gamma^{\prime}+\gamma^{\prime \prime}$. Given that the three terms in the second line are equivalent, the contribution of them in calculations is the same. Thus, by using (2.12), the $\mathcal{S}_{\gamma \rightarrow \gamma^{\prime}+\gamma^{\prime \prime}}$ is obtained as: the door to measuring the gravitational effects on quantum fields which can be measured in the laboratory scale of energy. We postpone this direct calculation to next works, and in this work, we calculate only the null curvature limit of (3.6) for comparing with Minkowski results.

\section{Scattering matrix in null curvature limit}

In flat space-time, the interaction Hamiltonian between the electromagnetic field and spinor field is $\mathcal{H}_{\text {int }}=e \bar{\psi} A^{A} \psi$ [30-33, 42]. The $\mathcal{S}$-matrix for $\lambda=3$ in Minkowski space is:

$$
\begin{aligned}
\mathcal{S}^{(3)} & =\frac{i}{3 !} \int \mathrm{d}^{4} X_{1} \int \mathrm{d}^{4} X_{2} \int \mathrm{d}^{4} X_{3} T\left[\mathcal{H}_{\mathrm{int}}\left(X_{1}\right) \mathcal{H}_{\mathrm{int}}\left(X_{2}\right) \mathcal{H}_{\mathrm{int}}\left(X_{3}\right)\right] \\
& =\frac{i e^{3}}{6} \int \mathrm{d}^{4} x_{1} \int \mathrm{d}^{4} X_{2} \int \mathrm{d}^{4} X_{3} T\left[(\bar{\psi} \not A \psi)_{X_{1}}(\bar{\psi} A \bar{\psi})_{X_{2}}(\bar{\psi} \not \bar{\psi} \psi)_{X_{3}}\right] .
\end{aligned}
$$

Fig. 1 Interaction of three photons diagrams

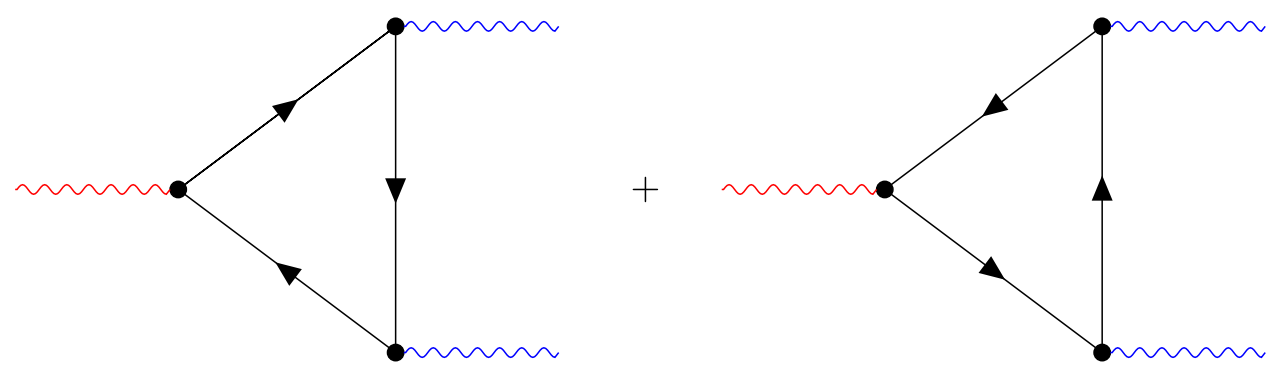


By using Wick theorem and definition of spinor two-point function [30], similar to that came in Section III, the $\mathcal{S}^{(3)}$ for interaction of three photons is:

$$
\begin{aligned}
\mathcal{S}_{\left(\gamma \rightarrow \gamma^{\prime}+\gamma^{\prime \prime}, \gamma^{\prime}+\gamma^{\prime \prime} \rightarrow \gamma\right)}^{(3)} & \\
= & \mathcal{S}_{a}^{(3)}+\mathcal{S}_{b}^{(3)}=-\frac{e^{3}}{6 \hbar^{3}} \int \mathrm{d}^{4} X_{1} \int \mathrm{d}^{4} x_{2} \int \mathrm{d}^{4} X_{3} \\
& \times\left\{N\left[A\left(X_{1}\right) S\left(X_{1}, X_{2}\right) \not A\left(X_{2}\right) S\left(X_{2}, X_{3}\right) A\left(X_{3}\right) S\left(X_{3}, X_{1}\right)\right]\right. \\
& \left.+N\left[A\left(X_{1}\right) S\left(X_{1}, X_{3}\right) \not A\left(X_{3}\right) S\left(X_{3}, X_{2}\right) A\left(X_{2}\right) S\left(X_{2}, X_{1}\right)\right]\right\} .
\end{aligned}
$$

As it is seen, these two terms are the same and similar to the previous section one can consider only one of them. So by using vector field operator, spinor two-point function [30-33, 42] and delta function definition in flat space-time, after some mathematical calculations, the $\mathcal{S}^{(3)}$ for interaction $\left(\gamma \longrightarrow \gamma^{\prime}+\gamma^{\prime \prime}\right)$ is obtained as follows:

$$
\begin{aligned}
\mathcal{S}_{\left(\gamma \longrightarrow \gamma^{\prime}+\gamma^{\prime \prime}\right)}^{(3)=} & i \int \mathrm{d}^{3} k \int \mathrm{d}^{3} k^{\prime} \int \mathrm{d}^{3} k^{\prime \prime} \\
& \sum_{r} \sum_{r^{\prime}} \sum_{r^{\prime \prime}} \chi d^{r}(k) d^{\dagger r^{\prime}}\left(k^{\prime}\right) d^{\dagger r^{\prime \prime}}\left(k^{\prime \prime}\right),
\end{aligned}
$$

where

$$
\begin{aligned}
\chi & =-i e^{3} \mathcal{N}_{k} \mathcal{N}_{k^{\prime}} \mathcal{N}_{k^{\prime \prime}} \int \mathrm{d}^{4} p \int \mathrm{d}^{4} p^{\prime} \int \mathrm{d}^{4} p^{\prime \prime} \delta^{4}\left(p^{\prime}\right. \\
& -p+k) \delta^{4}\left(p^{\prime \prime}-k^{\prime}-p^{\prime}\right) \delta^{4}\left(p-p^{\prime \prime}-k^{\prime \prime}\right) \\
& \times\left\{\frac{\operatorname{Tr}\left[(\not p+m) \notin^{(r)}(k)\left(\not p^{\prime}+m\right) \notin^{\left(r^{\prime}\right)}\left(k^{\prime}\right)\left(\not p^{\prime \prime}+m\right) \notin^{\left(r^{\prime \prime}\right)}\left(k^{\prime \prime}\right)\right]}{\left(p^{2}-m^{2}+i \tau\right)\left(p^{\prime 2}-m^{2}+i \tau\right)\left(p^{\prime \prime 2}-m^{2}+i \tau\right)}\right\} .
\end{aligned}
$$

Given that the trace of odd number of gamma matrices is zero, with respect to the Delta function property, one can obtain this relation:

$$
\begin{aligned}
& \chi=-i e^{3} \mathcal{N}_{k} \mathcal{N}_{k^{\prime}} \mathcal{N}_{k^{\prime \prime}} \quad \delta^{4}(k \\
& \left.-k^{\prime}-k^{\prime \prime}\right) \int \mathrm{d}^{4} p \\
& \frac{1}{\left[p^{2}-m^{2}+i \tau\right]\left[(p-k)^{2}-m^{2}+i \tau\right]\left[\left(p-k^{\prime \prime}\right)^{2}-m^{2}+i \tau\right]} \\
& \times\left\{\operatorname{Tr}\left[\not p \notin \not p \notin^{\prime} \not p \notin^{\prime \prime}\right]-\operatorname{Tr}\left[\not p \notin \not p \notin^{\prime} \not k^{\prime \prime} \notin^{\prime \prime}\right]\right. \\
& -\operatorname{Tr}\left[\not p \notin \not k \notin^{\prime} \not p \notin^{\prime \prime}\right]+\operatorname{Tr}\left[\not p \notin \not k \notin^{\prime} \not k^{\prime \prime} \notin^{\prime \prime}\right] \\
& +m^{2} \operatorname{Tr}\left[\not p \notin \notin^{\prime} \notin^{\prime \prime}\right] \\
& +m^{2} \operatorname{Tr}\left[\notin \not \not \notin^{\prime} \notin^{\prime \prime}\right]+m^{2} \operatorname{Tr}\left[\notin \notin^{\prime} \not \not \notin^{\prime \prime}\right] \\
& \left.-m^{2} \operatorname{Tr}\left[\notin \not k \phi^{\prime} \notin^{\prime \prime}\right]-m^{2} \operatorname{Tr}\left[\notin \notin^{\prime} \not k^{\prime \prime} \notin^{\prime \prime}\right]\right\} \text {, }
\end{aligned}
$$

where $\epsilon \equiv \epsilon^{(r)}, \epsilon^{\prime} \equiv \epsilon^{\left(r^{\prime}\right)}, \epsilon^{\prime \prime} \equiv \epsilon^{\left(r^{\prime \prime}\right)}$. The energy of outgoing photons is the same, and it is equal to half the energy of incoming photons, $k_{0}^{\prime}=k_{0}^{\prime \prime}=\frac{1}{2} k_{0}$. By attention to $k^{2}=0$ and by supposing that the direction of outgoing photons is the same for simplicity, one can obtain:

$\vec{k}^{\prime}=\vec{k}^{\prime \prime}=\frac{1}{2} \vec{k}, \quad \longrightarrow \quad k^{\prime \mu}=k^{\prime \prime} \mu=\frac{1}{2} k^{\mu}$.

After some mathematical calculations, the constant $\chi$ is obtained as [43]:

$$
\begin{aligned}
\chi & =\frac{2}{3} \pi^{2} e^{3}(\gamma-1) \delta^{4}\left(k-k^{\prime}\right. \\
& \left.-k^{\prime \prime}\right) \mathcal{N}_{k} \mathcal{N}_{k^{\prime}} \mathcal{N}_{k^{\prime \prime}}\left[\left(k \cdot \epsilon^{\prime}\right)\left(\epsilon \cdot \epsilon^{\prime \prime}\right)-\left(k \cdot \epsilon^{\prime \prime}\right)\left(\epsilon \cdot \epsilon^{\prime}\right)\right],
\end{aligned}
$$

where the $\gamma=0.5772157$ is Euler's constant and one can write $\gamma=-\Gamma(0)-\lim _{n \rightarrow 0}(n)$ [44]. It is clear that the coefficient $\chi$ also depends on the energy momentum and the polarization states of the photons.

\section{Null curvature limit}

The dS manifold is a hyperboloid with radius $H^{-1}$. For large radius, or equivalently $H \rightarrow 0$, the curvature of space-time vanishes so the $H \rightarrow 0$ limit is called null curvature limit. In this limit, dS space-time matches with Minkowski space-time. The following coordinate system is useful in calculating the null curvature limit:

$$
\begin{gathered}
x^{\alpha} \equiv\left(H^{-1} \sinh \left(H X^{0}\right), \frac{\vec{X}}{H\|\vec{X}\|} \cosh \left(H X^{0}\right) \sinh (H\|\vec{X}\|)\right. \\
\left.H^{-1} \cosh \left(H X^{0}\right) \cosh (H\|\vec{X}\|)\right)
\end{gathered}
$$

where $\|\vec{X}\|=\left(X_{1}^{2}+X_{2}^{2}+X_{3}^{2}\right)^{\frac{1}{2}}$. By using this coordinate system, it is easy to show that the $\mathrm{dS}$ flat waves of massive fields $(x \cdot \xi)^{-2 \pm i v},(x \cdot \xi)^{-1 \pm i v}$ or $(x \cdot \xi)^{\frac{3}{2} \pm i v}$ in null curvature limit reduce to Minkowski flat wave $e^{ \pm i k \cdot X}$ [16]. Also for massless vector fields, the dS flat wave $(x \cdot \xi)^{-2}$ or $(x \cdot \xi)^{-1}$ can be mapped to intrinsic coordinate and then it can be shown that the null curvature limit of them matches with their Minkowskian counterparts [16, 34]. Also, one can show that [16]:

$\lim _{H \rightarrow 0} H \not y=\lim _{H \rightarrow 0} H \eta_{\alpha \beta} \gamma^{\alpha} x^{\beta}=-\gamma^{4}$.

For dS massless vector field, one can show that the polarization states are $[16,34]$ : 
$\lim _{H \rightarrow 0} \mathcal{E}_{2 \alpha}(x, \xi, n)=\lim _{H \rightarrow 0} \mathcal{E}_{1 \alpha}(x, \xi, n)=\epsilon_{\mu}^{(n)}$,

where $\mu=0,1,2,3$ and $\epsilon_{\mu}^{(n)}$ is the polarization of massless vector field in Minkowski space-time. By attention to Dirac equation for spinor (2.5) and adjoint spinor (2.8), one can see that the null curvature limits of $\Psi$ and $\bar{\Psi}$ are:

$\lim _{H \rightarrow 0} \Psi(x)=\psi(X), \quad \lim _{H \rightarrow 0} \bar{\Psi}(x) \gamma^{4}=\bar{\psi}(X)$.

Thus, by attention to (5.1) and (2.9), one can obtain:

$$
\begin{aligned}
\lim _{H \rightarrow 0} \mathbf{S}\left(x_{1}, x_{2}\right) & =-i \lim _{H \rightarrow 0}\left\langle\Omega\left|\Psi\left(x_{1}\right) \bar{\Psi}\left(x_{2}\right)\right| \Omega\right\rangle \\
& =i\left\langle 0\left|\psi\left(X_{1}\right) \bar{\psi}\left(X_{2}\right) \gamma^{4}\right| 0\right\rangle=-S\left(X_{1}, X_{2}\right) \gamma^{4} .
\end{aligned}
$$

Therefore, one can obtain the null curvature limit of $\chi^{\mathrm{d} S}$ as:

$$
\begin{aligned}
\lim _{H \rightarrow 0} \chi^{\mathrm{d} S}= & -\frac{i q^{3}}{\hbar^{3}} \mathcal{N}_{k} \mathcal{N}_{k^{\prime}} \mathcal{N}_{k^{\prime \prime}} \\
& \iiint \mathrm{d}^{4} X_{1} \mathrm{~d}^{4} X_{2} \mathrm{~d}^{4} X_{3} e^{-i k \cdot X_{1}} e^{-i k^{\prime} \cdot X_{2}} e^{-i k^{\prime \prime} \cdot X_{3}} \\
& \times \operatorname{Tr}\left\{\gamma^{4}\left(-\gamma^{4}\right) \epsilon_{\mu} \gamma^{\mu}\left(S\left(X_{1}, X_{2}\right) \gamma^{4}\right)\right. \\
& \left.\gamma^{4}\left(-\gamma^{4}\right) \epsilon_{\nu} \gamma_{\nu}\left(S\left(X_{2}, X_{3}\right) \gamma^{4}\right) \gamma^{4}\left(-\gamma^{4}\right) \epsilon_{\lambda} \gamma^{\lambda}\left(S\left(X_{3}, X_{1}\right) \gamma^{4}\right)\right\},
\end{aligned}
$$

By using (2.4) and Fourier transformation of spinor twopoint function $S\left(X, X^{\prime}\right)$, and also by attention to that $\gamma^{4} \gamma^{4}=-I$, one can obtain:

$$
\begin{aligned}
\lim _{H \rightarrow 0} \chi^{\mathrm{d} S} & =\frac{1}{(2 \pi)^{12}} \frac{i q^{3}}{\hbar^{3}} \mathcal{N}_{k} \mathcal{N}_{k^{\prime}} \mathcal{N}_{k^{\prime \prime}} \iiint \mathrm{d}^{4} p \mathrm{~d}^{4} p^{\prime} \mathrm{d}^{4} p^{\prime \prime} \\
& \frac{\operatorname{Tr}\left[\theta(\not p+m) \theta^{\prime}\left(\not p^{\prime}+m\right) \theta^{\prime \prime}\left(\not p^{\prime \prime}+m\right)\right]}{\left(p^{2}-m^{2}+i \tau\right)\left(p^{\prime 2}-m^{2}+i \tau\right)\left(p^{\prime \prime 2}-m^{2}+i \tau\right)} \\
& \times \iiint \mathrm{d}^{4} X_{1} \mathrm{~d}^{4} X_{2} \mathrm{~d}^{4} X_{3} \\
& \left\{e^{-i k \cdot X_{1}} e^{i k^{\prime} \cdot X_{2}} e^{i k^{\prime \prime} \cdot X_{3}} e^{-i p \cdot\left(X_{1}-X_{2}\right)} e^{-i p^{\prime} \cdot\left(X_{2}-X_{3}\right)} e^{-i p^{\prime \prime} \cdot\left(X_{3}-X_{1}\right)}\right\},
\end{aligned}
$$

where, $\notin=\epsilon_{\mu} \gamma^{\prime \mu}$. After some mathematical calculations, the $\lim _{H \rightarrow 0} \chi^{\mathrm{d} S}$ is obtained as:

$$
\begin{aligned}
& \lim _{H \rightarrow 0} \chi^{\mathrm{d} S}=\frac{i q^{3}}{\hbar^{3}} \mathcal{N}_{k} \mathcal{N}_{k^{\prime}} \mathcal{N}_{k^{\prime \prime}} \delta^{4}\left(k-k^{\prime}-k^{\prime \prime}\right) \\
& \quad \times \int \mathrm{d}^{4} p^{\prime \prime} \frac{\operatorname{Tr}\left[(\not p+m) \theta(\not p-\not k+m) \theta^{\prime}\left(\not p-\not k^{\prime \prime}+m\right) \theta^{\prime \prime}\right]}{\left(p^{2}-m^{2}+i \tau\right)\left((p-k)^{2}-m^{2}+i \tau\right)\left(\left(p-k^{\prime \prime}\right)^{2}-m^{2}+i \tau \tau\right)} .
\end{aligned}
$$

As it seen, the null curvature limit of $\mathcal{S}_{\gamma \rightarrow \gamma^{\prime}+\gamma^{\prime \prime}}^{(3)}$ is matched on Minkowski result (4.3) exactly.

\section{Conclusion}

A photon decaying to two photons in $\mathrm{dS}$ ambient space formalism is investigated, and its $\mathcal{S}$-matrix elements are obtained in the first approximation. Indirect gravitational effect appears in this $\mathcal{S}$-matrix elements from the two-point functions and the polarization vectors. This gravitational effect can be calculated explicitly by numerical methods which may be done in the next work. It was shown that the $\mathcal{S}$-matrix elements in the null curvature limit exactly reduce to the Minkowskian counterparts.

The Hamiltonian interaction of three photons was obtained from quantum field theory approach. The interaction constant $\chi$ is approximately obtained theoretically. This constant depends on the energy momentum and the polarization states of photons. These photons interact with electrons of the spinor loop or virtual electrons which appear in the quantum vacuum fluctuations. It should be noted that these electrons are not those of the matter or crystal, these are electrons that create and annihilate in quantum vacuum fluctuations that interact with the real photons. These results are obtained for one incoming photon, and we ignore the bulk effects. The bulk effects could be investigated in the next works.

Acknowledgements The authors wish to express their particular thanks to A. Rabeei, M. Amiri, M. Rastiveis and R. Razani for useful discussions.

Open Access This article is distributed under the terms of the Creative Commons Attribution 4.0 International License (http://creativeco mmons.org/licenses/by/4.0/), which permits unrestricted use, distribution, and reproduction in any medium, provided you give appropriate credit to the original author(s) and the source, provide a link to the Creative Commons license, and indicate if changes were made.

\section{References}

1. Rottwitt, K., Tidemand-Lichtenberg, P.: Nonlinear Optics. CRC Press, Boca Raton (2010)

2. Fox, M.: Quantum Optics an Introduction. Oxford University Press, Oxford (2006)

3. Walls, D.F., Milburn, G.J.: Quantum Optics. Springer, Berlin (2008)

4. Garrison, J.C., Chiao, R.Y.: Quantum Optics. Oxford University Press, Oxford (2008)

5. Ralph, T.C., Taubman, M.S., White, A.G., McClelland, D.E., Bachor, H.A.: Squeezed light from second-harmonic generation: experiment versus theory. Opt. Lett. 20, 11 (1995)

6. Grynberg, G., Aspect, A., Fabre, C.: Introduction to Quantum Optics. Cambridge University Press, Cambridge (2010)

7. Bachor, H.A., Ralf, T.C.: A Guide to Experiments in Quantum Optics. Wiley, New York (2004)

8. Jalilifard, F., Ahmadi, Y., Takook, M.V.: Electron-photon interaction in de Sitter ambient space formalism. Mod. Phys. Lett. A 33, 1850006 (2017)

9. Ahmadi, Y., Jalilifard, F., Takook, M.V.: Scalar-spinor fields interaction in de Sitter ambient space formalism. Mod. Phys. Lett. A (2019). https://doi.org/10.1142/S0217732319502055

10. Perlmutter, S., et al.: Measurement of $\Omega$ and $\Lambda$ from 42 highredshift supernovae. Astrophys. J. 517, 565 (1999)

11. Riess, A.G., et al.: Observational evidence from supernovae for an accelerating universe and a cosmological constant. Astron. J. 116, 1009 (1998). [arXiv:9805201v1] 
12. de Bernardis, P., et al.: A flat universe from high-resolution maps of the cosmic microwave background radiation. Nature 404, 955 (2000)

13. Henry, J.P., Briel, U.G., Bohringer, H.: The evolution of galaxy clusters. Sci. Am. 279, 52 (1998)

14. Henry, J.P.: Measuring cosmological parameters from the evolution of cluster X-ray temperatures. Astrophys. J. 534, 565 (2000). [arXiv:0002365]

15. Ade, P.A.R., et al.: Detection of B-mode polarization at degree angular scales by BICEP2. Phys. Rev. Lett. 112, 241101 (2014). [arXiv:1403.3985v3]

16. Takook, M.V.: Théorie quantique des champs pour des systèmes élémentaires "massifs" et de "masse nulle" sur l'espace-temps de de Sitter. Thèse de l'université Paris VI (1997)

17. Takook, M.V., Azizi, A., Babaian, E.: Covariant quantization of massive spin- $-\frac{3}{2}$ fields in the de Sitter space. Eur. Phys. J. C 72, 20206 (2012). [arXiv:1206.1997]

18. Behroozi, S., Rouhani, S., Takook, M.V., Tanhayi, M.R.: Conformally invariant wave equations and massless fields in de Sitter spacetime. Phys. Rev. D 74, 124014 (2006). [arXiv:gr-qc/05121 05]

19. Enayati, M., Rouhani, S., Takook, M.V.: Quantum linear gravity in de Sitter universe On Gupta-Bleuler vacuum state. Int. J. Theor. Phys. 56, 1068 (2017). [arXiv:1208.5562v1]

20. Takook, M.V.: Spin $\frac{1}{2}$ field theory in the de Sitter space-time. In: Proceedings of the Group 21, 15-20 July, Goslar, Germany. [arXiv :gr-qc/0005077] (1996)

21. Dehghani, M., Rouhani, S., Takook, M.V., Tanhayi, M.R.: Conformally invariant massless spin-2 field in the de Sitter universe. Phys. Rev. D 77, 064028 (2008). [arXiv:0805.2227]

22. Takook, M.V.: Quantum field theory in de Sitter universe: ambient space formalism. [arXiv:1403.1204] (2016)

23. Tanhayi, M.R., Mirabi, S.: Linear energy-momentum tensor for a scalar field in de Sitter space (2012). https://doi. org/10.1186/2251-7235-6-24

24. Mehdizadeh, K., Jalili, O.: Charged particles in curved spacetime. https://doi.org/10.1007/s40094-015-0200-x (2015)

25. Dehghani, M.: Conformal graviton two-point function in de Sitter space. https://doi.org/10.1186/2251-7235-6-44 (2012)

26. Fatahi, N.: Conformally invariant spin $3 / 2$ field equation in de Sitter space-time. [https://doi.org/10.1007/s40094-015-0189-1] (2015)

27. Bartesaghi, P., Gazeau, J.P., Moschella, U., Takook, M.V.: Dirac fields and thermal effects in de Sitter universe. Class. Quantum Grav. 18, 4373 (2001)
28. Moradi, S., Rouhani, S., Takook, M.V.: Discrete symmetries for spinor field in de Sitter space. Phys. Lett. B 613, 74 (2005). [arXiv :gr-qc/0502022]; [Erratum, Phys. Lett. B 658284 (2008)]

29. Bros, J., Moschella, U.: Two-point functions and quantum fields in de Sitter universe. Rev. Math. Phys. 8, 327 (1996). [arXiv:grqc/9511019]

30. Mandl, F., Shaw, G.: Quantum Field Theory. Wiley, New York (2010)

31. Kaku, M.: Quantum Field Theory. Oxford University Press, Oxford (1993)

32. Itzykson, C., Zuber, J.B.: Quantum Field Theory. McGraw-Hill, New York (1980)

33. Ryder, L.: Quantum Field Theory. Cambridge University Press, Cambridge (1996)

34. Garidi, T., Gazeau, J.P., Rouhani, S., Takook, M.V.: Massless vector field in de Sitter universe. J. Math. Phys. 49, 032501 (2008). [arXiv:gr-qc/0608004]

35. Takahashi, B.: Sur les représentations unitaires des groupes de Lorentz généralisés. Bull. Soc. Math. France 91, 289 (1963)

36. Gazeau, J.P., Takook, M.V.: Massive vector field in de Sitter space. J. Math. Phys. 41, 5920 (2000). [arXiv:gr-qc/9912080]

37. Garidi, T., Gazeau, J.P., Takook, M.V.: Comment on: Massive vector field in de Sitter space. J. Math. Phys. 43, 6379 (2002)

38. Rouhani, S., Takook, M.V.: Abelian gauge theory in de Sitter space. Mod. Phys. Lett. A 20, 2387 (2005). [arXiv:gr-qc/0502019]

39. Takook, M.V.: Quantum de Sitter-black hole in ambient space formalism. In: Lecture Presented at 5th Workshop and Seminar on Topics in Theoretical Physics. Azarbaijan Shahid Madani University, 23-25 August. [arXiv:1711.01067] (2017)

40. Floratos, E.G., Iliopoulos, J., Tomaras, T.N.: Tree-level scattering amplitudes in de Sitter space diverge. Phys. Lett. B 197, 373 (1987)

41. Antoniadis, I., Iliopoulos, J., Tomaras, T.N.: One-loop effective action around de Sitter space. Nucl. Phys. B 462, 437 (1996)

42. Weinberg, S.: The Quantum Theory of Fields. I. Cambridge University Press, Cambridge (1995)

43. Ahmadi, Y., Takook, M.V.: The Hamiltonian of three photons interaction. Mod. Phys. Lett. A (2019) (under review)

44. Refaei, A.: Euler-Heisenberg lagrangian through Krein regularization. Int. J. Mod. Phys. A 28, 14 (2013) 